\title{
UNO STUDIO SUL TERREMOTO DEL GRAN SASSO D'ITALIA DEL 5 SETTEMBRE 1950
}

\author{
D. Di Filippo - L. Marcelli
}

Notizie macrosismiche. - 115 settembre 1950 , alle $5^{\mathrm{h}} 10^{\mathrm{m}}$ ca., un gran numero di persone aljitanti a Roma fu destata da una forte scossa di terremoto. Alcuni, specie se dimoranti nei piani più elevati dei palazzi, ne furono addirittura spaventati; e questo per Roma fu una novità, perché la città, pur essendo circondata dai Colli Laziali sovente interessati da lievi scosse telluriche, molto difficilmente le rileva in modo sensibile. Nei primi momenti ci fu un certo allarme; la curiositi poi prese il sopravento: il giornale radio delle prime ore non faceva menzione del fatto, in attesa ancora di notizie sicure.

Ùn primo esame (effettuato dal personale dell'Istituto Nazionale di Geofisica) dei sismogrammi della stazione sismica di Roma chiarì trattarsi di un terremoto con epicentro distante circa $90 \mathrm{~km}$. La violenza del sisma, invero noterole, aveva fatto uscire fuori scala tutte le immagini luminose degli strumenti a registrazione ottica: quanto ai sismografi a registrazione meccanica, nei più sensibili erano uscite fuori sede le pennine: si dové lavorare su quelli a scarsa sensibilità (il $200 \mathrm{~kg}$ orizzontale Wiechert e l'80 $\mathrm{kg}$ verticale Wiechert), sebhene anche una delle due pennine del $200 \mathrm{~kg}$ fosse stata sbalzata fuori sede dalla violenza delle onde superficiali. Si riuscì ad individuare la prohabile zona epicentrale come situata a N-E di Roma, nei pressi del Monte Pozzon, alle pendici del Gran Sasso d'Italia. È nota la difficoltà di determinare, con una sola stazione, un epicentro; tanto più poi in questo caso giacché la scossa era stata preceduta da un'altra, di minore intensitì, le cui onde superficiali non si erano ancora estinte al sopragrgiungere della seconda, più violenta. Tuttavia tale determinazione risultò confermata dalle prime notizie macrosismiche che cominciarono a pervenire.

In breve anche la radio e la stampa furono in grado di dare ampi resoconti delle località più colpite. Pauroso bilancio invero, reso più tragico dalla recente guerra che con i bombardamenti di pochissimi anni fa ha reso malsicure le case ed assolutamente inadatte a resi- 
stere ad un terremoto. Teramo, Pescara, Rieti, L'Aquila risultarono le quattro province più fortemente danneggiate. L'intensità della scossa all'epicentro e per una vasta zona circostante fu ralutata di VIII della scala Mercalli. Danni gravi si lamentarono a Campli, Tossicia, Isola del Gran Sasso, Celino Attanasio, Penne; tutti paesi in provincia di Teramo, dove crollarono complessivamente 64 case e 600 rimasero danneggiate più o meno gravemente: a Pietracamela (Teramo) tutte le case furono lesionate e gli abitanti costretti all'aperto. A Farindola (Pescara) due contadini morirono sotto il crollo della loro casetta. Oltre un centinaio di persone furono ferite più o meno gravemente. Nei pressi di Amatrice (Rieti) una persona dichiarò di aver udito un boato profondo e di aver veduto vampate rosse e viola immediatamente prima di sentirsi tremare la terra sotto i piedi.

In provincia di Ascoli Piceno 410 fabbricati furono dichiarati inabitabili dal Genio Civile. Altri rombi furono sentiti più o meno forti a Civitaquana, Isola del Gran Sasso, Fano Adriano, Accumoli, Pizzoli e in molte altre localiti. Ad Amatrice e Scoppito fu notato un forte vento durato alcune ore. A Farindola le acque entrarono in movimento come fossero in ebollizione, a Montereale l'acqua nelle sorgenti subì un sensibile aumento, a Castelli fu notato un precipitar di massi dalla vicina montagna.

Queste le notizie macrosismiche più importanti, interessanti l'immediata area epicentrale, parte ricavate dalla stampa e parte gentilmente forniteci dall'Lifficio Centrale di Meteorologia.

La fig. 1 mostra la fotografia di una casa gravemente lesionata.

Isosiste. - Nella fig. 2 abbiamo tracciato la rete delle linee isosiste così come si son potute disegnare dopo uno scrupoloso esame delle notizie macrosismiche pervenuteci. Risulta così che la scossa è stata sentita di grado VIII nelle seguenti localiti: Arsita, Colledara, Castelli, Isola del Gran Sasso, Cellino Attanasio, Bisenti, Fano Adriano, Pietracamela, Tossicia (Teramo), Elice, Farindola, Civitaquana (Pescara), Montereale, Arischia, Pizzoli (Aquila), Amatrice, Accumoli (Rieti); di VII-VIII a Scoppito (Aquila) e Borbona (Rieti). E stato avvertito di VII ad Antrodoco, Cittaducale, Posta, Micigliano (Rieti); Alanno, Civitella del Tronto, Loreto Aprutino, Rocciano, Montesilvano, Penne, Pianella (Pescara); Paganica, Barisciano, San Demetrio, Fossa (Aquila); Montegallo, Venarotta, Palmiano, Arquata del Tronto, Roccafluvione (Ascoli Piceno); Norcia (Perugia); di VI-VII a Bo- 
lognano e Toccocasauria (Pescara); Nereto (Teramo); di VI a Pescara, Salle, Caramanico, Cepagatti, Popoli (Pescara); Tione degli Abruzzi, Rocea di Cambio, Rocca di Mezzo, Navelli (Aquila); Piediluco, Arrone (Terni); Controguerra, Colonnella, Pineto (Teramo); Concerviano, Labro, Marcetelli (Rieti); Visso (Macerata); Offida, Comunanza e di V-VI a Ripatransone (A. scoli Picenol. Nelle seguenti località invece $\grave{e}$ stato sentito di V: Anversa degrli Abruzzi, Pescina, Gioia dei Marsi, Pet-

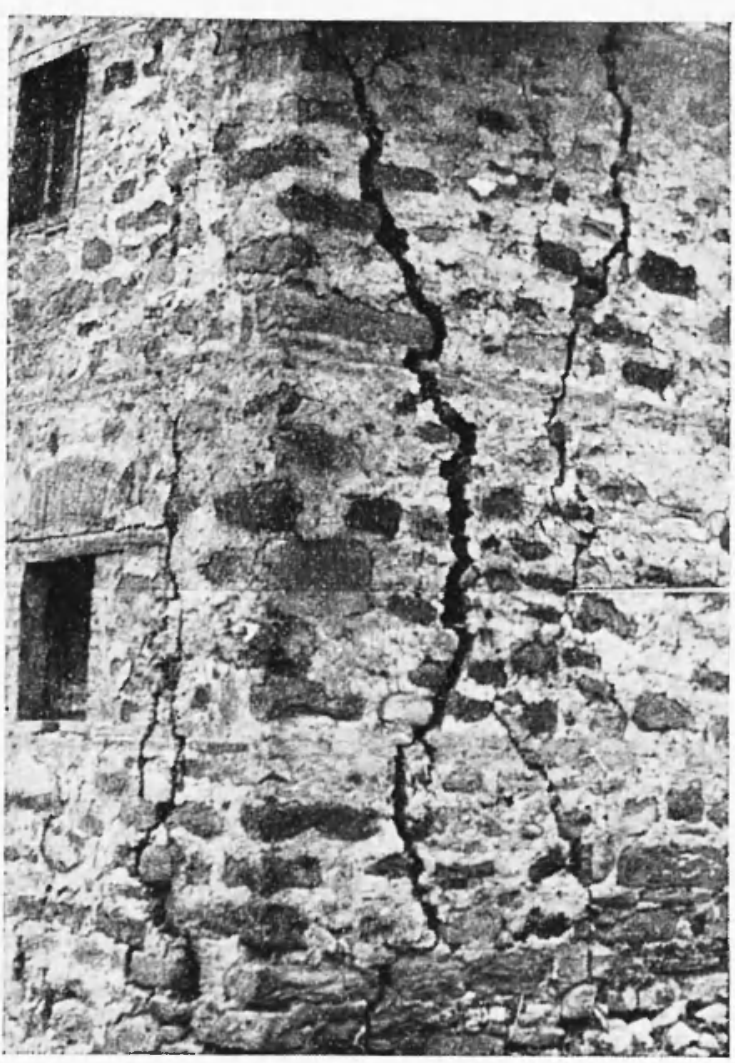

Fig. 1 torano sul Gizio, Magliano dei Marsi, Carsoli, Raiano, Pacentro, Goriano Siculi, Sulmona, Pratola (Aquila); Stimigliano, Nespolo, Petescia, Toffia, Castelnuovo di Farla, Casperia, Forano (Rieti); Macerata, Tolentino, Bolognola, Matelica, Montecassiano (Macerata); Foligno, Nocera, Spoleto, Campello sul Clitunno. Spello, Montefalco (Perugia); Terni, Narni (Terni); Fermo (Ascoli Piceno) e di IV-V a Rocca Pia, Capistrelli, Scanno (Aquila), Cannara (Perugia); di IV a Jesi, Fabriano, Ancona, Staffolo, Agugliano, Genga, Ostra, Barbara, Loreto e Camerano (Ancona); Perugia, Assisi (Perugia); Monteleone dOrvieto (Terni); Roma, Subiaco (Roma); Ronciglione e Montefiascone (Viterbo); Rivisondoli, Roccaraso (Aquila); Filettino (Frosinone); di IlI-IV a: Ripe, Sinigallia (Ancona); Tuscania (Viterbo); Città di Castello (Perugia); di III a: Frosinone, Fiuggi, Ferentino, Guarcino, Ceprano, Alatri, Arpino, Sarteano, Casamari, Sora (Frosinone); Velletri e di II a Priverno (Roma). 
D. DI FILIPl' - L. MARCFLLI

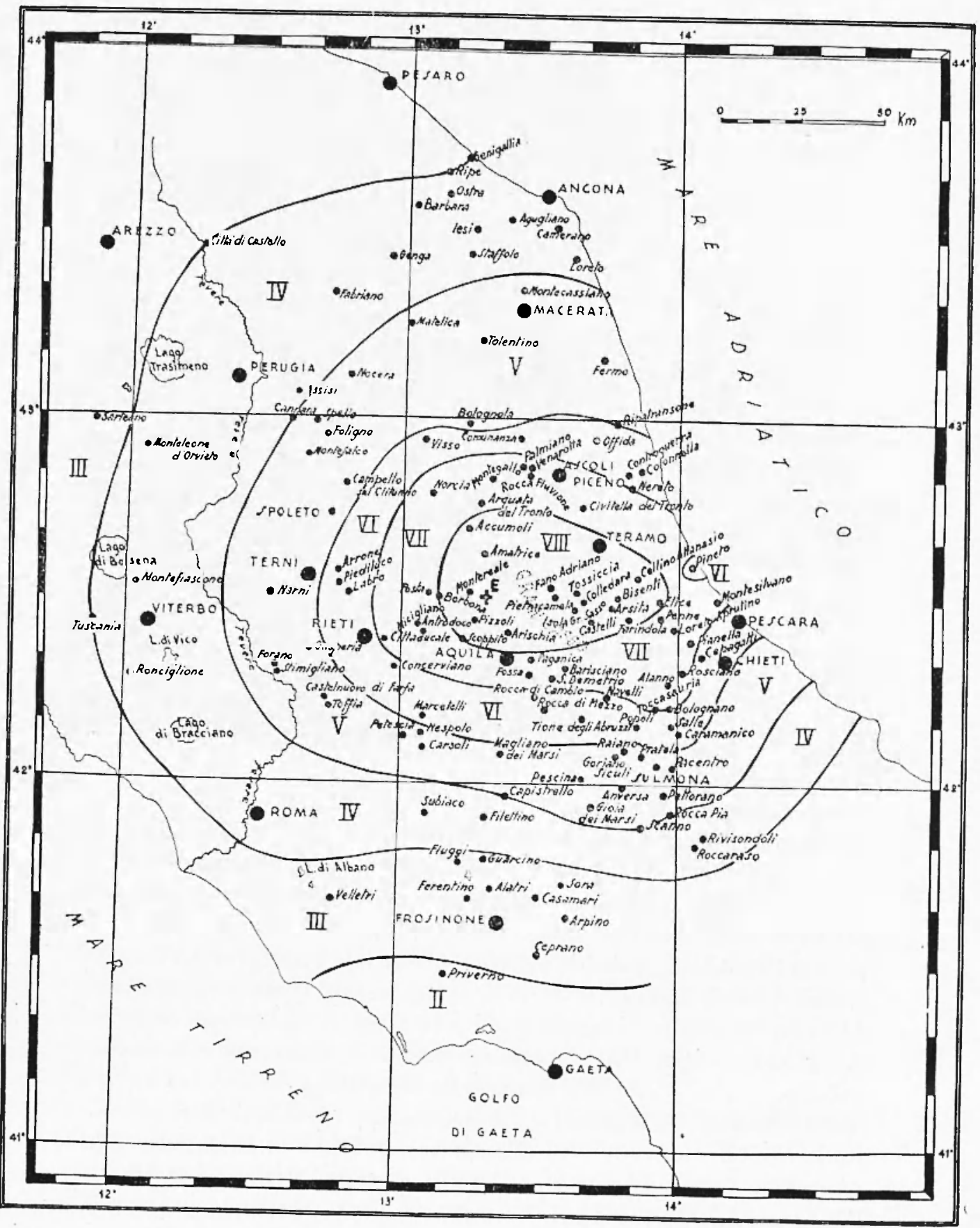

Fig. 2 
La violenza del sisma si è estinta solo dopo circa $150 \mathrm{~km}$ dalla zona epicentrale.

La distriluzione delle isosiste non è molto regolare, specialmente se si osserva la posizione dell'epicentro: la più forte anomalia si presenta ad est e a sud-est di esso, e questo, molto probalbilmente, data la vicinanza del massiccio del Gran Sasso d'Jtalia e dato inoltre che, come si vedrà, il terremoto è molto superficiale, può trovare la sua spiegazione nella struttura geologica della zona.

Tale anomalia è messa maggiormente in evidenza nella figura 3 dove, alla rete delle isosiste reali $\grave{e}$ sorrapposta una rete di isosiste circolari i cui raggi sono stati calcolati facendo le medie aritmetiche - su una distriluzione radiale di 10 in 10 gradi - delle distanze epiecntrali $\perp$ rela-

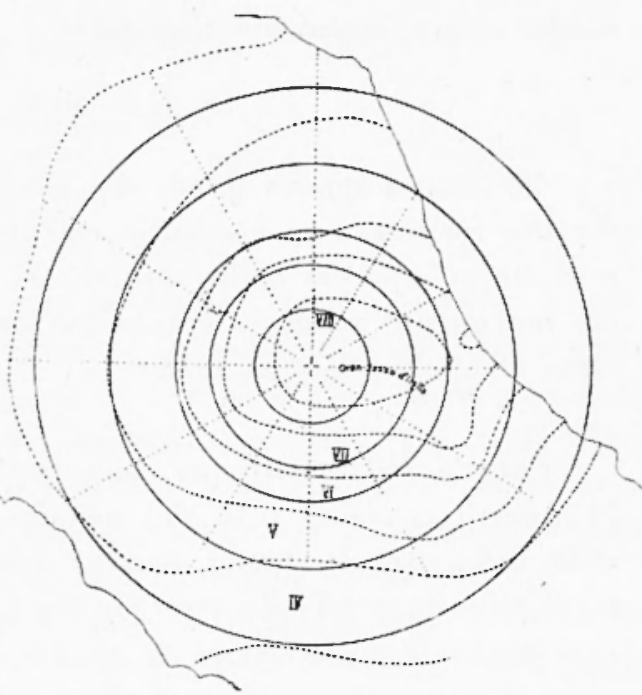

Fig. 3 tive ad una stessa isosista, secondo la formula data da Sieberg $\left({ }^{1}\right)$.

$$
R_{\mathrm{i}}=\frac{\Delta_{1}+\Delta_{2}+\Delta_{3}+\cdots+\Delta_{n}}{n}
$$

Risultereble in tal modo che il raggrio della zona epicentrale (VIII Mercalli) è di $24 \mathrm{~km}$, mentre in realtà ci sono dei punti distanti fino a $55 \mathrm{~km}$.

I raggi delle isosiste circolari successive sono i seguenti:

$R_{v 11}=41 \mathrm{~km} ; R_{v_{1}}=54 \mathrm{~km} ; R_{\mathrm{r}}=80 \mathrm{~km} ; R_{\mathrm{tv}}=111 \mathrm{~km} ; R_{111}=119 \mathrm{~km}$.

Magnitudo ed energia del terremoto. - Valendoci della determinazione effettuata in 5 stazioni italiane (Roma, Bologna, Firenze Arcetri, Palora, Saliol albsiamo calcolato la Magnitudo del terremoto con le formule valide in ciascuna di dette stazioni $\left({ }^{2}\right)$. Si è così trovato un valore di 5,45 . La scossa infatti è stata registrata fino ad Helsinki, in Finlandia (oltre $2000 \mathrm{~km}$ distante dall'epicentro), ed i 
sismografi di stazioni molto più lontane, come Tucson (oltre 10.000 $\mathrm{kmi}$ ne hanno rilevato l'inizio.

L'energia associata a questo terremoto, calcolata con la formula ( $\left.{ }^{2}\right)$

$$
\log E=9,154+2,147 M
$$

risulta essere, approssimativamente,

$$
E \sim 10^{21} \text { ergs }
$$

Notiamo a questo punto che questo terremoto è stato preceduto da una forte scossa, e seguito, nel giro di poche ore, da numerose repliche di intensitì molto minore, talché l'energia sviluppata in questo movimento tellurico risulta ovviamente superiore a quella associata alla sola scossa in esame.

Calcolo delle coordinate epicentrali. - Per la determinazione dell'epicentro ci siamo valsi del metodo Caloi basato sulla conoscenza delle differenze dei tempi d'arrivo delle onde $\mathrm{Pg}$ ed $\mathrm{Sg}\left({ }^{3}\right)$ nelle stazioni più vicine all'epicentro. Questo metodo ci ha permesso di sfruttare anche le registrazioni di alcune stazioni vicine sfortunatamente prive, in quel griorno, di correzione del tempo.

È noto in che cosa consista tale metodo: ci limitiamo pertanto a riportarne le formule essenziali.

Si abbiano a disposizione $n$ stazioni. Siano $x_{0}$ e $y_{0}$ le coordinate ortogonali dell'epicentro, $x_{\mathrm{i}} y_{\mathrm{i}}$ le coordinate ortogonali della $i^{m a}$ stazione $(i=1,2, \ldots n)$. Valgono allora le seguenti equazioni che cost $\vec{i}-$ tuiscono un sistema il quale andrà risolto con il metodo dei minimi quadrati

$$
\left(x_{\mathrm{i}}-x_{\mathbf{o}}\right)^{2}+\left(y_{\mathrm{i}}-y_{\mathbf{o}}\right)^{2}=\left(K T_{\mathrm{i}}\right)^{2}
$$

essendo

$$
T_{\mathrm{i}}=\left(t_{2}-t_{1}\right)_{\mathrm{i}}
$$

dove $t_{1}$ e $t_{2}$ sono i tempi di registrazione delle onde $P g$ ed $S g$ rispettivamente, in una stessa stazione, e $K$ ha il seguente valore

$$
K=\frac{v_{1} \cdot v_{9}}{v_{1} \cdot v_{2}},
$$

$v_{1}$ e $v_{2}$ essendo le velocità delle onde longitudinali e trasversali dirette. 
Se si riferiscono tutte le stazioni ad una (la prima) scelta come fondamentale, le [1] si scrivono, risolte rispetto ad $x_{0}, y_{0}$

$$
x_{0}+\frac{y_{j}-y_{1}}{x_{j}-x_{1}} y_{0}-\frac{c_{j}-c_{1}}{x_{j}-x_{1}}=0 \quad(j=2,3, \ldots n)
$$

dove le $c$ hanno il valore dato dalla espressione

Se poniamo

$$
2 c_{\mathrm{j}}=-\left(K T_{\mathrm{j}}\right)^{2}+x^{2}{ }_{\mathrm{j}}+y^{2}{ }_{\mathrm{j}}
$$

$$
\alpha=\frac{y_{\mathrm{j}}-y_{1}}{x_{\mathrm{j}}-x_{1}} \quad ; \quad \beta=\frac{c_{\mathrm{j}}-c_{1}}{x_{\mathrm{j}}-x_{1}}
$$

le [3] si possono scrivere

$$
x_{\mathrm{o}}+\alpha y_{\mathrm{o}}=\beta
$$

Nell'applicare questo metodo ci si serve, come si è detto, di coordinate ortogonali. A questo proposito va notato (t) che per la determinazione di dette coordinate non ci si deve riferire ad un meridiano e ad un parallelo considerati come assi ortogonali $y$ e $x$. Il riferimento va fatto ad un meridiano (asse $y$ ) e ad un cerchio massimo ad esso ortogonale (asse $x$ ), che non va confuso con il parallelo passante per il punto d'incrocio dei due cerchi massimi considerati.

Per maggiori dettagli rimandiamo all'opera citata ( $\left.{ }^{+}\right)$. Qui ci limitiamo a trascrivere le formule finali di cui ci siamo serviti per il nostro lavoro.

Indichiamo con t. e $\varphi$ le coordinate geografiche di una qualsiasi stazione $S$, con $\lambda_{4}$ e $r_{0}$ le coordinate geografiche del punto $O$ di riferimento; si avrà che le coordinate ortogonali $x, y$ di detta stazione $S$ avranno le seguenti espressioni:

$$
\left\{\begin{array}{l}
x=r_{\mathrm{o}}\left[\frac{\Delta \lambda}{\alpha^{\prime \prime}} \cos \varphi-\frac{(\Delta \lambda)^{3}}{6 \alpha^{\prime \prime 3}} \cos \varphi \sin ^{2} \varphi\right] \\
y=r_{\mathrm{o}}\left[\frac{\varphi-\varphi_{0}}{\alpha^{\prime \prime}}+\frac{1}{4}-\left(\frac{\Delta \lambda}{\alpha^{\prime \prime}}\right)^{2} \sin 2 r^{\prime}\right]
\end{array}\right.
$$

dove $\Delta \lambda=\lambda-\gamma_{0}, \quad r_{0}$ è il raggio della Terra supposta sferica ed $\alpha^{\prime \prime}=206265$. Se però si ruol tener conto della eccentricità della Terra, le [7] vanno scritte come segue:

$$
\left\{\begin{array}{l}
x=r_{\mathrm{n}}\left[\frac{\Delta \lambda}{\alpha^{\prime \prime}} \cos \varphi-\frac{(\Delta \lambda)^{3}}{6 \alpha^{\prime \prime 3}} \cos \varphi \sin ^{2} \varphi\right] \\
y==r_{\mathrm{m}}\left[\frac{\varphi-\Upsilon_{0}}{\alpha^{\prime \prime}}+\frac{1}{4}\left(\frac{\Delta \lambda}{\alpha^{\prime \prime}}\right)^{2} \sin 2 \varphi\right] .
\end{array}\right.
$$




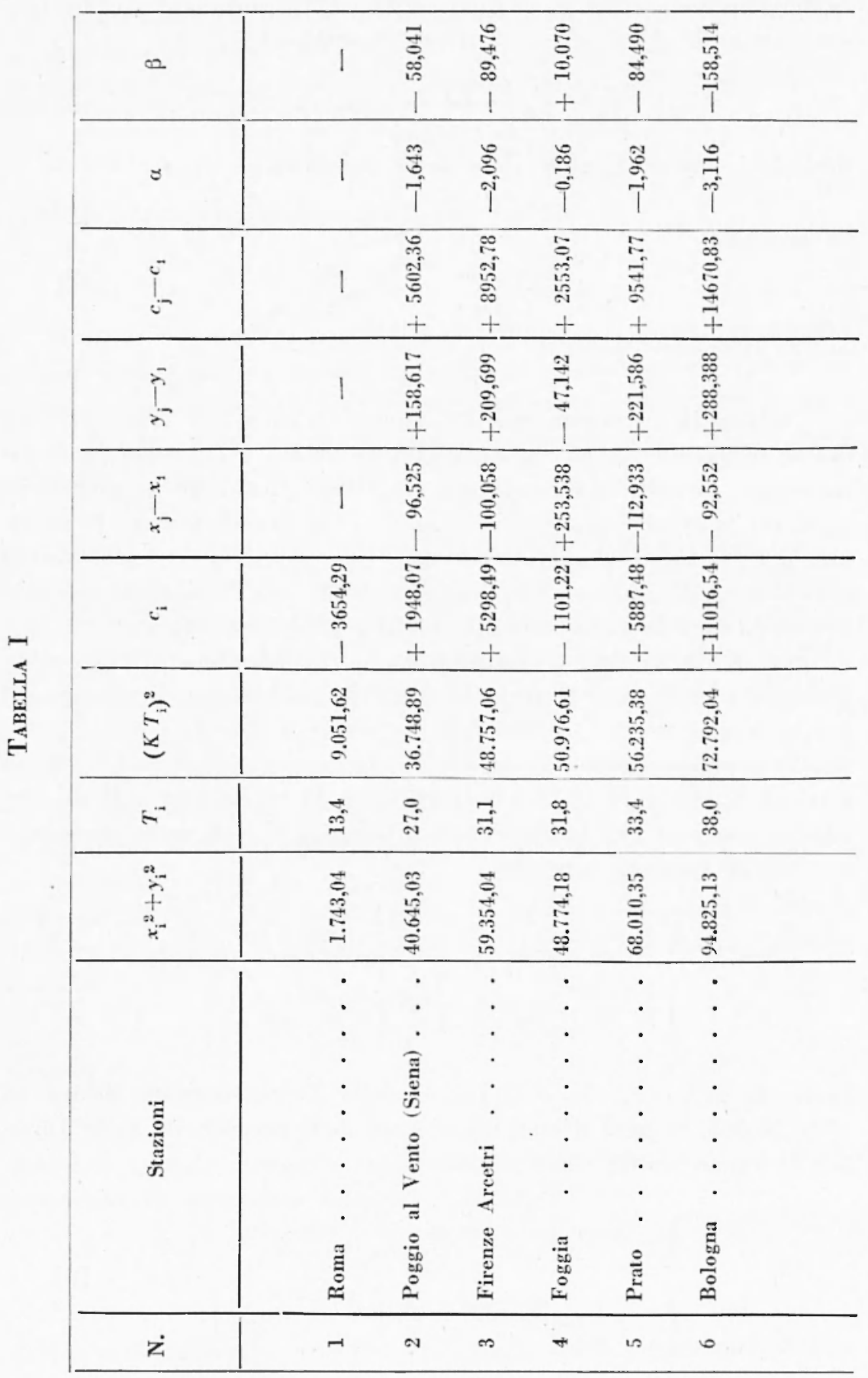


Se chiamiamo $\left(y_{1}\right.$ la latitudine della proiezione di $S$ sopra il meridiano fondamentale, grandezza legata alle altre dalla relazione

$$
p_{1}=\varphi_{0}+\alpha^{\prime \prime} \frac{y}{r_{0}} \quad
$$

nella prima delle [8] $r_{n}$ rappresenta il raggio della sfera osculatrice dell'ellissoide lungo il parallelo di latitudine $\varphi_{1}$; nella seconda, $r_{\mathrm{m}}$ rappresenta il corrispondente raggio di curvatura del meridiano: $r_{n}$ va riferito a $\varphi_{0}, r_{m}$ va riferito a $\frac{\varphi_{1}+\varphi_{0}}{2}$

Nel caso del presente lavoro, data la posizione dell'epicentro del nostro terremoto, abbiamo assunto i seguenti valori:

$$
\left\{\begin{array}{l}
\varphi_{0}=42^{\circ} \mathrm{N} . \\
\lambda_{0}=13^{\circ} \mathrm{E} .
\end{array}\right.
$$

Per il calcolo degli $r_{n}$ ed $r_{m}$ relativi all'ellissoide di Bessel ci siano valsi della tabella che compare nell'opera citata.

Fra i molti sismogrammi messi gentilmente a nostra disposizione da quasi tutti gli osservatori europei, abbiamo scelto, per questa prima determinazione epicentrale, quelli tra i più vicini, che avevano una registrazione ben chiara: alcune stazioni erano prive della correzione del tempo ma questo, dato il metodo che applichiamo, non ha importanza. Riportiamo, a titolo di esempio, le registrazioni ottenute a Roma (sull'80 kg verticale Wiechert), e a Chur (figg. 4-5). Nella tabella che segue sono esposti i risultati dei calcoli per la determinazione delle coordinate ortogonali delle stazioni scelte. I valori delle $x_{i}, y_{i}$, sono ottenuti applicando le [8].

1. Roma

2. Poggrio al Vento (Siena)

3. Firenze Arcetri

4. Fograia

5. Prato

6. Bologna

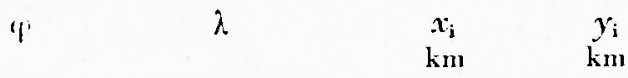

$$
41^{\circ} 54^{\prime}, 2 \quad \mathrm{~N} \quad 12^{\circ} 30^{\prime}, 8 \quad \mathrm{E}-4.0,3764-10,6203
$$$$
43^{\circ} 19^{\prime}, 2 \text { » } 11^{\prime \prime} 18^{\prime}, 71 \text { ” }-136,9010+147,9971
$$$$
43^{\circ} 45^{\prime}, 73 \text { ) } 11^{\circ} 15^{\prime}, 33 \text { ) - } 140,4343+199,0785
$$$$
41^{\circ} 27^{\prime}, 1 \text { » } 15^{\circ} 33^{\prime}, 1 \text { ” }+213,1614-57,7623
$$$$
43^{\circ} 53^{\prime} \text { " } 11^{\circ} 05^{\prime}, 5 \text { " }-153,3097+210,9656
$$$$
44^{\circ} 29^{\prime}, 3 \text { » } 11^{\circ} 19^{\prime}, 7 \text { » }-132,9284+277,7682
$$

Per pervenire al sistema di equazioni [6] che ci permetterà di calcolare le coordinate ortogonali $x_{0} y_{0}$ dell'epicentro, abbiamo dovuto 
trevare $i$ valori delle $c_{j}$ serrendoci delle [4]. Ivi abbiamo assunto per $K$ il valore 7,1 , il dhe equivale a considerare la velociti delle $P g$ fari a $5.1 \mathrm{~km} / \mathrm{sec}$, e quella delle $S \underline{g}$ pari a $3,07 \mathrm{~km} / \mathrm{sec}$.

Abbiamo dapprima risolto il problema considerando tutle le stazioni alla stessa strequa: però i risultati a cui siamo pervenuti non

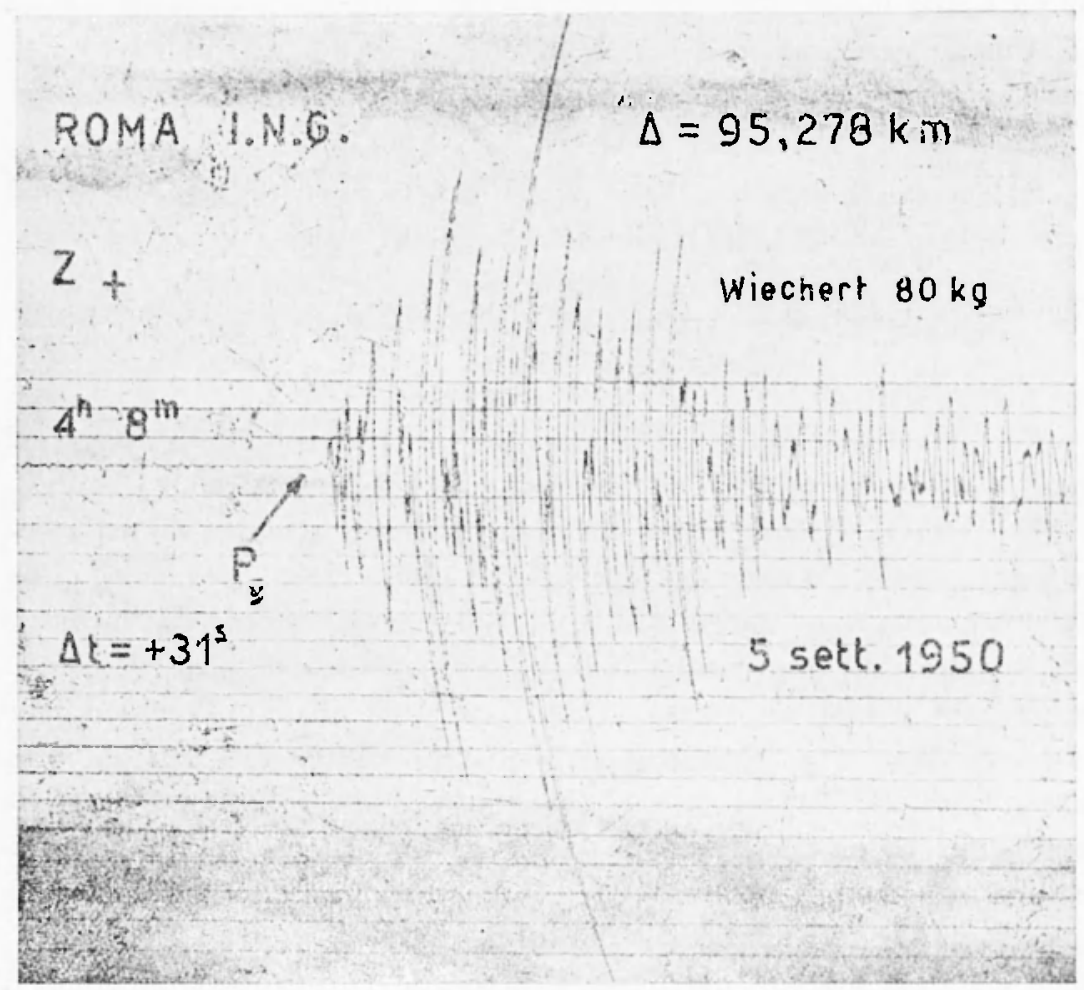

Fig. 4

erano troppo soddisfacenti, e abhiamo quindi preferito sceqlicre Roma come stazione fondamentale, essendo la più vicina all'epicentro.

Il sistema cui si perviene dopo aver applieato il metodo dei minimi quadrati alle $[6]$ è

$$
\left\{\begin{aligned}
5 x_{0}-9,003 y_{0} & =-330.151 \\
-9,003 x_{0}+20,686 y_{0} & =910,729
\end{aligned}\right.
$$


da cui si hamno i sequenti valori per le coordinate ortogonali dell'epicentro (in $\mathrm{km}$ )

$$
\left\{\begin{array}{l}
x_{0}=26,784 \\
y_{0}=57,133
\end{array}\right.
$$

Per passare ora alle coordinate geografiche, occorre far uso delle seguenti formule geodetiche di trasformazione $\left({ }^{t}\right)$ :

$$
\left\{\begin{array}{l}
\varphi_{\mathrm{r}}=\left(\varphi_{\mathrm{o}}+\alpha^{\prime \prime} \frac{y_{\mathrm{o}}}{r_{\mathrm{m}}}\right)-u^{\prime \prime} \frac{x_{\mathrm{o}}^{2}}{2{r_{\mathrm{n}}}^{2}} \lg \psi_{\mathrm{o}} \\
\lambda_{\mathrm{s}}=\lambda_{\mathrm{o}}+\alpha^{\prime \prime} \frac{x_{\mathrm{o}}}{r_{\mathrm{u}} \cos \left(\varphi_{\mathrm{o}}+\alpha^{\prime \prime} \frac{y_{\mathrm{o}}}{r_{\mathrm{m}}}\right)}
\end{array}\right.
$$

Si hamno i segrtenti risultati

$$
\left\{\begin{array}{l}
f_{E}=42^{\circ} 30^{\prime}, 8+2^{\prime}, 0 \mathrm{~N} \\
i_{E}=13^{\prime} 19^{\prime}, 6 \div 5^{\prime}, 4 \mathrm{E}
\end{array}\right.
$$

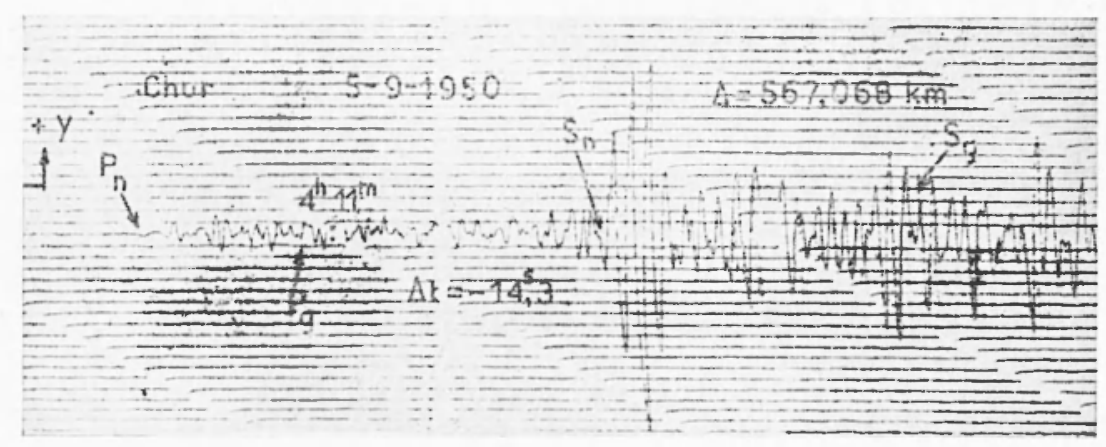

Fig. 5

Felociti di propugazione " dromocrone. - Conosciute così le coordinate geografiche dellepicentro, usando la nota formula di trigonometria sferica

$$
\cos \Delta=\sin \varphi_{\mathrm{r}} \sin \varphi+\cos \varphi_{\mathrm{r}} \cos \varphi \cos \left(\lambda_{\mathrm{r}}-\lambda_{\mathrm{r}}\right)
$$

ahbiamo calcolato le distanze epicentrali di unte le stazioni adoperate.

Nella tabella II riportiamo accanto a ciascuna stazione la distanza epicentrale calcolata, i tempi d'arrivo delle onde longitudinali e trasversali (espressi in G.C.T.) e la natura dell'impulso iniziale. 


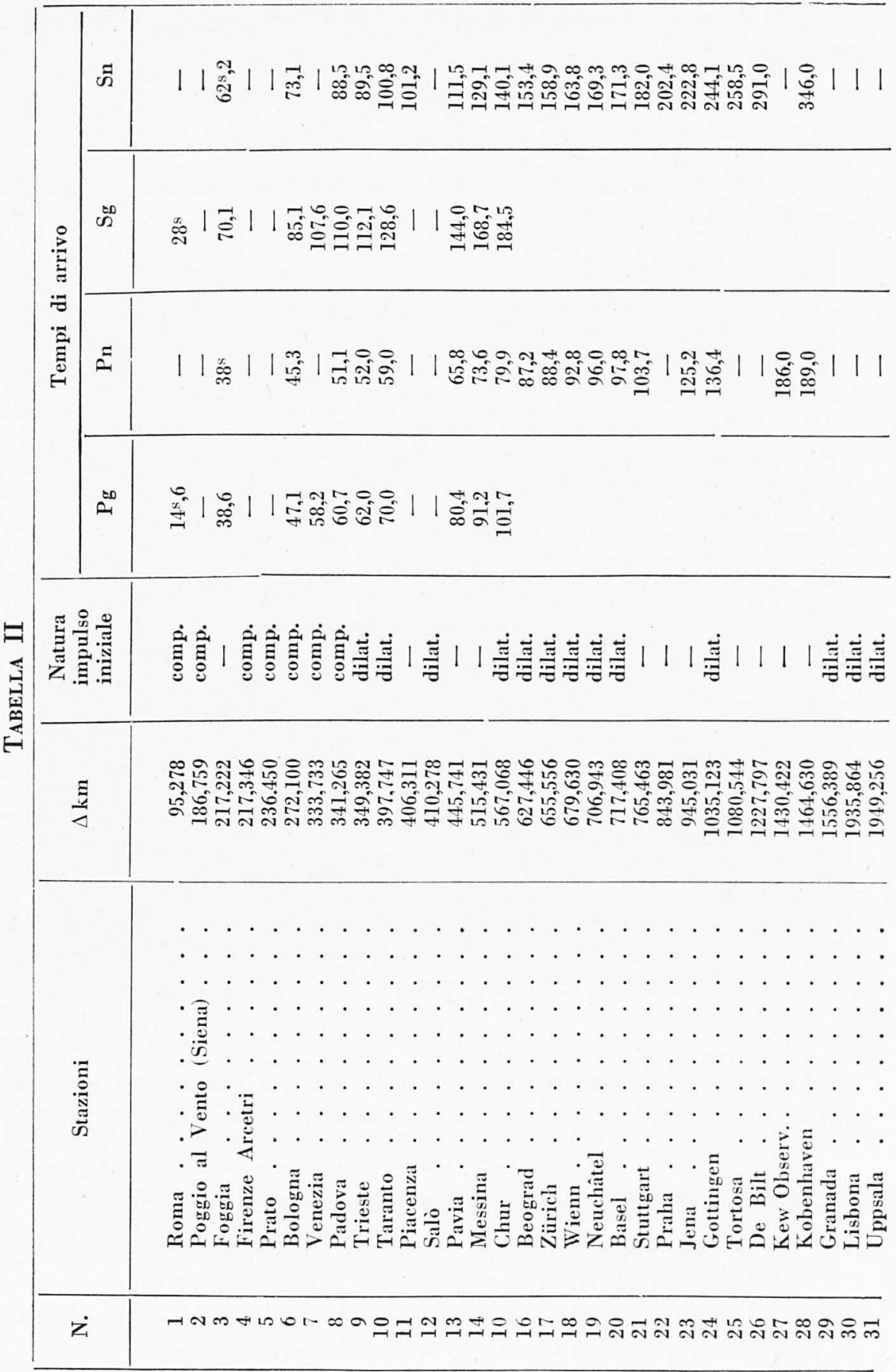


a) Onde Pg. - L'equazione di una generica dromocrona è, com'è noto, (per distanze relativamente piccole)

$$
t=\Delta y+x
$$

Nel nostro caso riferiamo la dromocrona ad un'ora origine prov. visoria del terremoto, che per comodità supponiamo

$$
04^{\mathrm{h}} 09^{\mathrm{m}} 00^{\mathrm{s}}
$$

Allora nella [13] $t$ rappresenta il tempo d'arrivo dell'onda in questione ad una stazione di distanza epicentrale $\Delta$. Scelte le 10 stazioni (Roma, Fogria, Bologna, Venezia, Padova, Trieste, Taranto, Pavia, Messina, Chur) le cui registrazioni presentavano ablastanza chiare le fasi $\mathrm{Pg}$ ed $\mathrm{Sg}$ abjuiamo risolto, col solito metodo dei minimi quadrati, il sistema e siamo griunti alla seguente equazione più probabile per la dromocrona delle $P g$ (riferita all'ora origine suddetta):

$$
t=(0,1831 \div 0,0018) \perp-2,27 \pm 0,69
$$

che può anche scriversi

$$
t=\frac{\Delta}{5,-46 \pm 0,18}-2,3 \div 0,7
$$

Di qui risulta una velocità di $5,46 \mathrm{~km} / \mathrm{sec}$. Il valore poco elevato di questa relocità trova tuttavia conforto in altri esempi. Già Caloi, sturiando un terremoto arvenuto nell'Appennino tosco-romarnolo ( ${ }^{5}$ ), aveva trovato per primo una $v_{1 \mathrm{~g}}=5,4 \mathrm{~km} / \mathrm{sec}$ : questo risultato, confermato successivamente da ulteriori studi, fa sì che il valore da noi trovato ¿̀ ormai quello accettato per l'Italia centrale.

Nella tabella III riportiamo gli scarti fra i tempi osservati e quelli calcolati con l'equazione ora scritta:

Tabella III

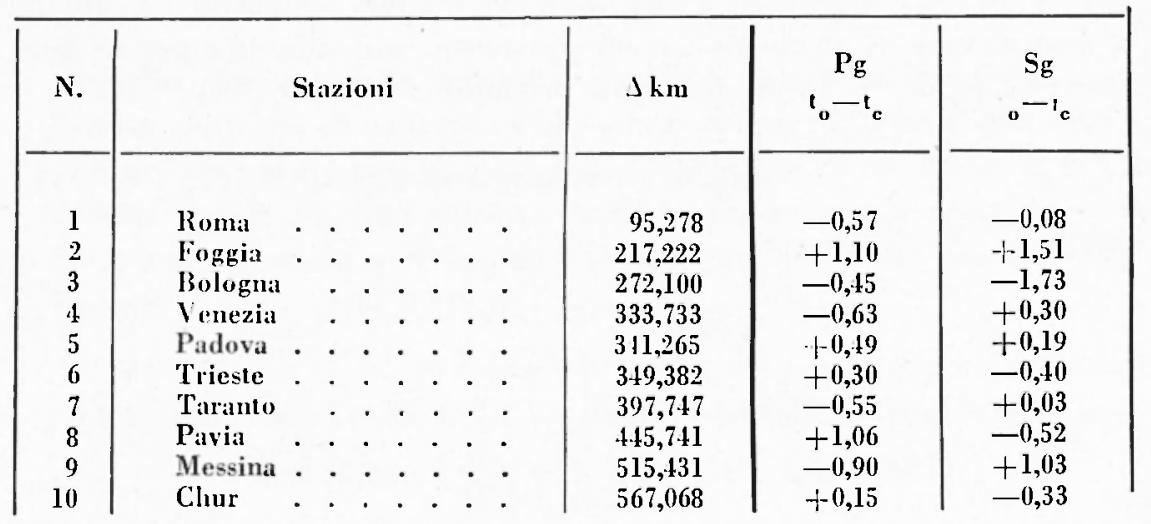


La somma dei quadrati degli scarti è $[v v]=4,72878$ che coincide praticamente con $[l l .2]=4,72880$, come i richiesto dalla teoria degli errori.

b) Onde $S_{z}-$ Con i tempi di arrivo delle $S g$ rilevati dalle stesse stazioni adoperate per le $P g$, abbiamo trovato che la dromocrona più attendibile per le onde $\mathrm{Sg}$ (riferita allo stesso tempo origine) risulta la seguente:

$$
t=(0,3322-0,0022) \Delta-3,6 \cdot+0,8
$$

oppure

$$
t=\frac{\Delta}{3,01-0,02}-3,6-0,8
$$

con una velocita, per dette onde, di $3,01 \mathrm{~km} / \mathrm{sec}$, valore che concorda perfettamente con quello trovato in precedenti ricerche (").

Nella tabella III sono riportati anche per le $S g$ gli scarti fra $i$ tempi osservati e quelli calcolati.

La somma dei quadrati degli scarti è

$$
[v v]=6,9773 \text { con }[l l .2]=6,9775 \text {. }
$$

Con i valori trovati per le velocità, ci siamo calcolati il valore del coefficiente di Poisson per lo strato del granito: esso é risultato $\sigma=0,28$ molto prossimo al valore medio trovato sperimentalmente.

c) Onde $P_{n}-$ Per studiare le onde $P_{n}$ ed $S_{n}$ ei siamo serviti, oviamente, di un maggior numero di stazioni (v. tabella II).

Anche qui riferiremo le dromocrone al tempo origine provvisorio 0.4 09m 00: Adoperati i dati delle 18 stazioni - scelte tra quelle a disposizione - il cui inizio era più netto, si è oltemnta per la dromocrona delle Pn l'equazione più probabile:

$$
t=(0,12205+0,00063 i \Delta+10,386-0,477
$$

oppure

$$
t=\frac{\Delta}{8,19-0,0 \cdot 4}-!(10,30+0,18)
$$

con una velocità delle $P$ n

$$
v_{\mathrm{r} \cdot \mathrm{u}}=8, \mathrm{i} 9 \cdot 0,04 \text {. }
$$


Nella tabella IV riportiamo gli scarti fra i tempi osservati e quelli calcolati. La somma dei quadrati deqrli scarti è:

coincidente con

$$
[v v]=13,7276 \text {, }
$$

$$
[l l .2]=13,7276 \text {. }
$$

II valore trovato per la velocità delle $P_{n}$ è leggermente superiore

\begin{tabular}{|c|c|c|c|c|c|c|c|c|c|}
\hline N. & Staz & ioni & & & & & $\Delta \mathrm{km}$ & $\begin{array}{r}\mathrm{Pg} \\
1-1\end{array}$ & $\begin{array}{l}S g \\
-1\end{array}$ \\
\hline 1 & Foggial & . & & & & & 217,222 & $+\mathbf{1}^{\mathrm{s}, 10}$ & $+2 s, 67$ \\
\hline 2 & Bologna & . & . & . & . & . & 272,100 & $+1,71$ & $+1,07$ \\
\hline 3 & Padova . & . & . & . & . & . & 311,265 & $-0,94$ & $+0,72$ \\
\hline 4 & Trieste . & . & . & . & . & • & 349,382 & $-1,03$ & $-0,13$ \\
\hline 5 & Taranto. & . & . & . & . & - & 397,747 & $+0,07$ & $+0,15$ \\
\hline 6 & Piacenza . & . & . & . & . & . & 106,311 & - & $-0,10$ \\
\hline 7 & Pavial . & . & . & . & . & • & $415,7.11$ & $+1,01$ & $-0,08$ \\
\hline 8 & Hessina. & . & . & . & . & . & 515,431 & $+0,31$ & $+1,65$ \\
\hline 9 & Chur . & . & . & . & . & . & 567,068 & $+(0,31$ & $+0,89$ \\
\hline 10 & Beograd & . & . & . & . & . & $627,4+16$ & $+0,24$ & $-0,43$ \\
\hline 11 & Ziiriclı . . & . & . & . & . & . & 655.556 & $-1,99$ & $+0,47$ \\
\hline 12 & Wienn : & . & . & . & & · & 679,630 & $-0,53$ & $-1,05$ \\
\hline 13 & Neuchatel . & . & . & . & & • & 706,943 & $-0,67$ & $-1,77$ \\
\hline 14 & Basel . . & . & . & . & & . & 717,408 & $-0,14$ & $-2,15$ \\
\hline 15 & Stuttgart & . & - & . & • & . & 765,463 & $-0,11$ & $-2,40$ \\
\hline 16 & Piahia & . & . & . & . & . & 843.981 & - & +0.11 \\
\hline 17 & Jena . . & . & $\cdot$ & . & - & 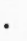 & $9\lfloor 5,031$ & $-0,52$ & $-2,50$ \\
\hline 18 & Gottingen. & . & . & . & . & - & 1035.123 & $-0,32$ & $-1,72$ \\
\hline 19 & Tortosia. & . & 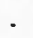 & . & - & . & $1080,5+4$ & - & $+2,33$ \\
\hline 20 & De Bilt & & 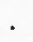 & . & • & - & 1227,797 & - & $+1,29$ \\
\hline 21 & Kew Obeerv & . & . & . & . & • & $1.430,422$ & $+1,0.4$ & - \\
\hline 22 & Kobenhaven & & . & . & 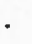 & . & $146 \cdot 1,630$ & $+0,46$ & +2.35 \\
\hline
\end{tabular}

\section{Tabella IV}

a quello commemente accettato. Tuttavia non mancano esempi, nell'Italia centrale, di velocità dello stesso ordine di grandezza: difatti, per Im terremoto con epicentro nella Toscana Settentrionale $\left({ }^{6}\right)$, fu trovata per le $P_{n}$ una velocità di $8,16 \mathrm{~km} / \mathrm{sec}$, e per un altro terremolo con epicentro nell'Appennino abruzzese ( ${ }^{\top}$ ) fu trovato $v_{l^{\prime} n}=8,25$ $\mathrm{km}$ sec.

d) Onde $S_{n}$ - L'equazione più probabile per la dromocrona delle Sn calcolata con i dati di 21 stazioni (tabella IJ) è risultata:

$$
t=(0,22777-0,00112)\lrcorner+(10,0522 \div 0,8383)
$$


che si può scrivere

$$
t=\frac{\Delta}{4,39-0,02}+(10,05 \div 0,84)
$$

La velocità delle $S_{n}$ risulta quindi:

$$
v_{\mathrm{sin}}=4,39+0,02
$$

La somma dei quadrati degli scarti (che sono elencali nella precedente tabella IV) ̀̀ $[v v]=51,2486$, essendo $[l l .2]=51,2410$.

Nella figura 6 riportiamo i grafici delle dromocrone trovate, riferite al tempo origine del terremoto.

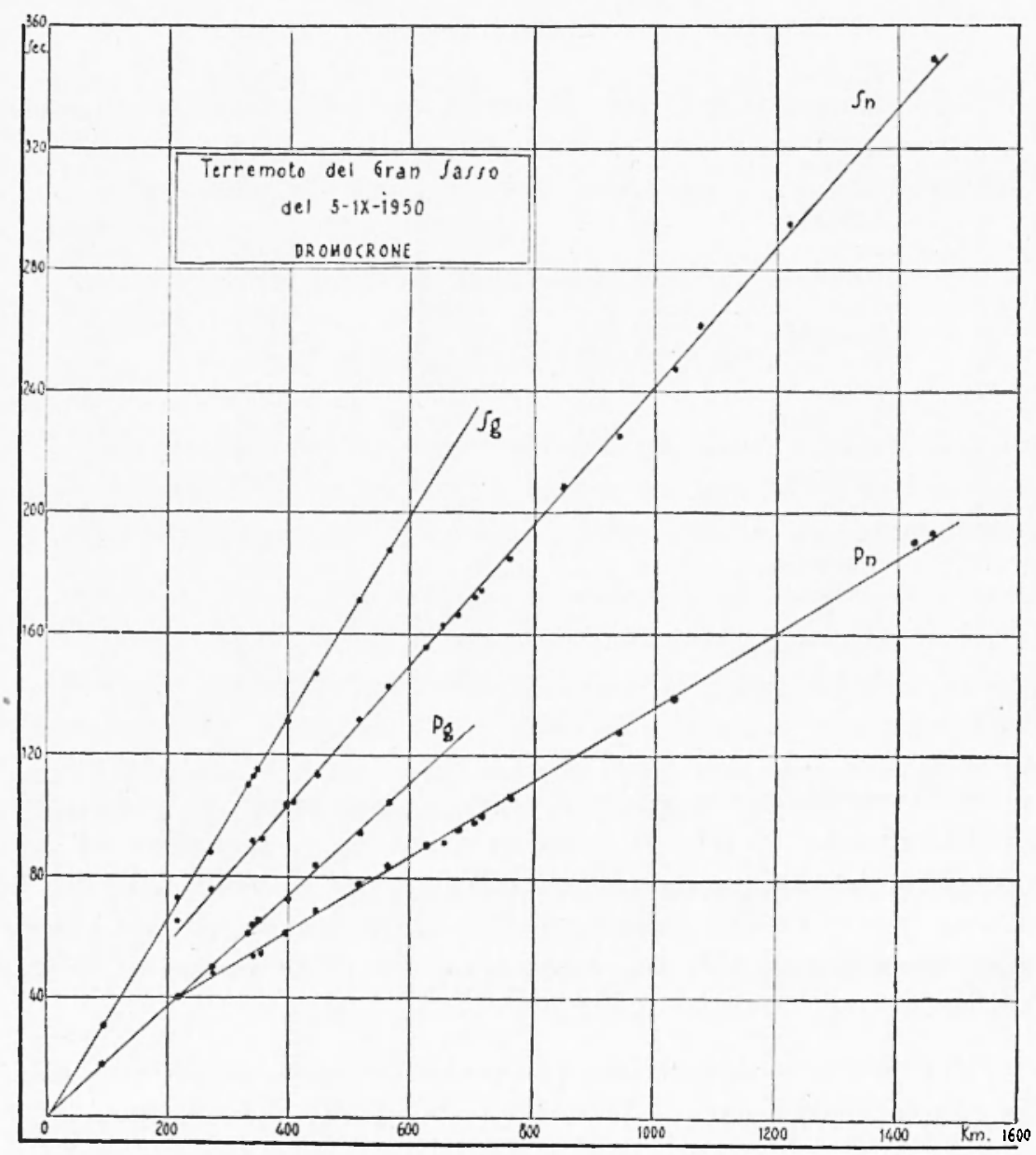

Fig. 6 
Nella tabella seguente riportiamo le velocità delle onde $P g, S g$, $P n, S n$, calcolate per l'Italia e per l'Europa centro-meridionale da vari ricercatori.

$\begin{array}{ccccc}P g & S g & P_{n} & S_{n}\end{array}$

1. Germania Merid. (Gutenberg)

5,55

-

8,21

2. Schwadorf (Conrad) . . .

5,6

3. Tirolo (Gräfe) . . . . . .

5,7

4. Prealpi Carniche (Caloi).

5,7

5. Cansiglio (Caloi) . . .

5,70

6. Alto Adriatico (Caloi) . . . -

7. Tosco Romagnolo (Caloi) . . 5,40

8. Garfagnana (Rosini) . . . .

9. Deruta (Genevois)

5,57 a Mord

5,25 a S.jd

5,43

3,39

8,12

$3,5 \quad 8,3$

3,3

3,36

7,81

8,00

- $\quad 7,77$

$3,0] \quad 7,86$

Sn

10. Monte Amiata 19 giumno 1940 (Di Filippo) .

5,6

3,30 a Murd 8,16

$(4,32)$

4,4

$\overline{4,41}$

4,8

4,34

4,44

Monte Amiata 16 ottolore $1940^{\circ}$

(Giorgi)

(Di ringos

5,16

12. Teramo (Di Filippo) . . .

13. Gran Sasso (Di Filippo-Marcelli)

14. Cervara di Roma (Di Filippo)

15. Ionio (Valle) . . . . . .

5,33

5,46

3,06 a sul -

3,25

5,39

$(6,05)$

3,26

7,8

3,02

3,20

$\overline{8,25}$

4,44

3,01

3,05

8,19

4,39

$(3,63) \quad 7,93$

4,40

4,57

Calcolo del tempo origine e della profondità ipocentrale. - Per il calcolo del tempo origine ci siamo serviti del metodo di Wadati e di quello di Inglada:

a) Metodo di Wadati: questo metodo, com'è noto $\left({ }^{8}\right)$, si basa sui tempi d'arrivo delle onde $P$ ed $S$ in parecchie stazioni prossime al. l'epicentro.

Scelto un sistema di assi ortogonali, su uno di questi si riportano i tempi di arrivo delle $P\left(T_{p}\right)$ (riferiti ad un valore base) e sull'altro le differenze tra i tempi d'arrivo delle $S$ e delle $P\left(T_{*-1}\right.$.. Si otterrà una retta che incontra l'asse delle $T_{\mathrm{p}}$ (quando è nulla la differenza $T_{\text {f-P: }}$ ) in un punto $H$ che è precisamente il tempo origine (riferito allo stesso valore).

Ahbiamo esteso il metodo allo strato del granito servendoci delle Pg ed Sg: scelte le 10 stazioni della tabella $\mathrm{V}$ e applicato, al solito, il metodo dei minimi quadrati, siamo pervenuti alla seguente equazione:

$$
0,62692+0,81234 T_{\mathrm{rk}}=T_{\mathrm{sg}-\mathrm{P}_{\mathrm{k}}}
$$

la quale, quando $T_{\mathrm{gg}-\mathrm{rg}}=0$ dà

$$
T_{\mathrm{ll}}=T_{\mathrm{l} \mathrm{B}}=-0^{*}, 7708
$$


Avendo riferito i calcoli al solito tempo $04^{\mathrm{h}} 09^{\mathrm{m}} 00^{\mathrm{s}}$ si arrebbe per $H$ il valore

$$
H=04^{\mathrm{h}} 08^{\mathrm{m}} 59^{\mathrm{s}}, 2
$$

b) Metodo di Inglada. Per avere una conferma del risultato conseguito, abbiamo applicato anche il metodo di Vicente Inglada ( ${ }^{9}$ ) valerole nel caso che si suppongano i raggi sismici rettilinei (abbastanza vero per piccole distanze epicentrali) propagantisi con velocità costante.

Vale allora la seguente espressione:

$$
\tau=\frac{1}{n-1} \stackrel{2}{2}_{2}^{n} \frac{\left(\Delta_{\mathrm{i}}^{2}-\Delta^{2}{ }_{\mathrm{i}-1}\right)-v^{2}\left(\tau_{\mathrm{i}}{ }^{2}-\mathrm{\tau}^{2}{ }_{\mathrm{i}-1}\right)}{2 v^{2}\left(\tau_{\mathrm{i}}-\tau^{2}{ }_{\mathrm{i}-1}\right)} \quad(i=1,2, \ldots n)
$$

dove $n$ è il numero delle stazioni, $\Lambda_{\mathrm{i}}$ la loro distanza epicentrale; $v$ è la velocità di propagazione del tipo d'onda prescelto, $\tau=t_{1}-t_{0}$, $\tau_{\mathrm{i}}=t_{\mathrm{i}}-\boldsymbol{t}_{t}, \quad\left(t_{\mathrm{i}}=\right.$ tempo d'arrivo delle onde $)$.

Eseguiti i calcoli con le 10 stazioni precedenti, assunto per $v_{\mathrm{I} g}$ il valore trovato di $5.46 \mathrm{~km} / \mathrm{sec}$ abbiamo ottenuto

$$
\tau=t_{1}-t_{0}=15^{\mathrm{s}}, 763
$$

ossia

Sicché sarì

$$
t_{0}=-1^{\mathrm{s}}, 163
$$

$$
H=04^{\mathrm{h}} 08^{\mathrm{m}} 58^{\mathrm{s}, 8}
$$

L'accordo tra i due risultati è abbastanza buono.

Se ora osserviamo le dromocrone delle onde $P^{\prime} g$ ed $S g$, deduciamo da esse che il tempo epicentrale è rispettivamente

$$
\begin{aligned}
& t_{\mathrm{s}}=04^{\mathrm{h}} 08^{\mathrm{m}} 57^{\mathrm{s}}, 7 \\
& t_{\mathrm{e}}=04^{\mathrm{h}} 08^{\mathrm{m}} 56^{\mathrm{s}}, 4
\end{aligned}
$$

1 vari metorli noti per il calcolo della profondit à ipocentrale per ter-

TABELLA $\dot{V}$

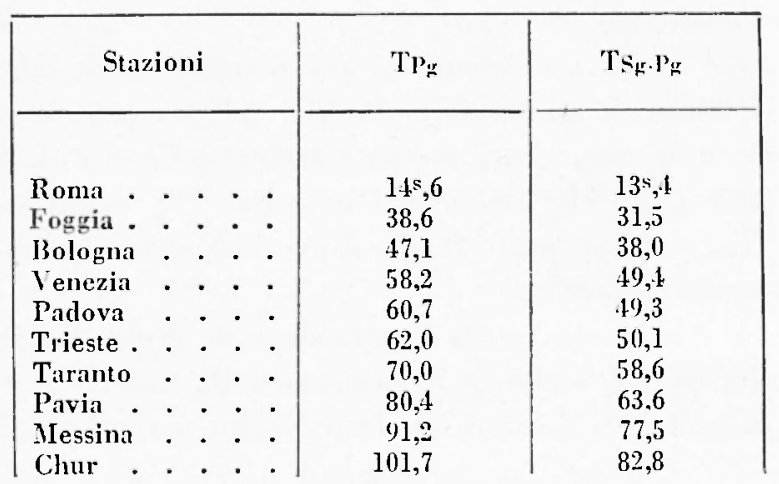
remoti vicini, non erano utilizzabili data la mancanza di stazioni molto vicine all'epicentro. Tuttavia adoperando la stazione di Roma 
(la più prossima) e applicanto la formula di Ingrada

alshiamo trovato

$$
h=\frac{\Delta^{2}-v_{\mathrm{Pg}}^{2}\left(t_{\mathrm{rg}}-t_{\mathrm{F}}\right)^{2}}{2 v_{\mathrm{P}^{\prime}}\left(t_{\mathrm{rg}^{\prime} \mathrm{g}}-t_{\mathrm{F}}\right)}
$$

$$
h=3,2 \mathrm{~km}
$$

La stessa formula, nel caso delle $S g$, ci lia dato

$$
h=0,2 \mathrm{~km}
$$

La $h$ dunque, risulta molto piccola. Si può affermare che essa è dell'ordine di qualche $\mathrm{km}$ (al di sotto dei 10 ), ma non se ne può pre. cisare il valore poiché si sa che quando $h$ è tanto piccola, tutti i metodi risentono, in modo decisivo, degli errori di osservazione, o strumentali, o delle piccole differenze nei tempi di propagazione delle onde sismiche.

L'epicentro coincide dunque praticamente con l'ipocentro. Il tempo origine, considerando i quattro valori trovati e facendone la media ponclcrale risulta cosi

$$
H=04^{\mathrm{h}}\left(08^{\mathrm{m}} 57^{\mathrm{s}, 5}-0^{\mathrm{s}, 5}\right.
$$

Notizie geologiche della regione epicentrale. - 11 teremoto in esame, come abbiamo visto, ha il suo epicentro addirittura in superficie. Il movimento, quindi, potreble avere una qualche relazione con la struttura geologica della zona. Può dunque presentare un certo interesse la conoscenza, sia pure superficiale, della tettonica della regrione epicentrale.

Osserviamo anzitutto che, esaminando la posizione dell'epicentro

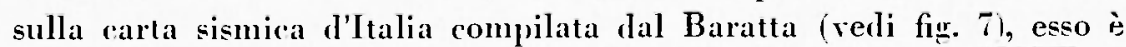
proprio al confine tra un'area di sismicita catastrofica i $\mathrm{N}-\mathrm{NW}$ ) e un'area di sismicità intensissima estendentesi, quest'ultima, ad Ovest e a Sud.

Geograficamente, l'epicentro i situato tra le sorgenti del fiume Aterno e del Vomano, sulle pendici nord-oceidentali del Gran Sasso d'Italia. Esso si trova quasi allineato sull'arco Abruzzese esterno, intendendo con tale denominazione l'allineamento anticlinale costituito dai Monti della Laga, il Gran Sasso d'Italia, la montagna del Morrone e la Majella (v. fig̣. 8).

I rilievi geologici di questa zona, dovuti a F. Sacco $\left({ }^{10}\right)$ sono incompleti nei dettagli: soltanto ora. per opera di J. Demangeot, ne è 
stato eseguito uno studio completo e minuzioso. Nella nostra esposizione ci riferiamo quindi, per lo più, all'opera di questo geologro (11).

Dal punto di vista stratigrafico, alla hase delle formazioni costituenti il gruppo montuoso in esame, è uno zoccolo semi-rigido di calcari compatti o brecciosi che vanno dal trias alloligocene incluso. La serie del giuralias è ben rappresentata, specialmente dal lias, molto potente, costituito prevalentemente di calcari dolomitici: la potenza del lias sembra raggiungere e forse oltrepassare anche i 500 metri

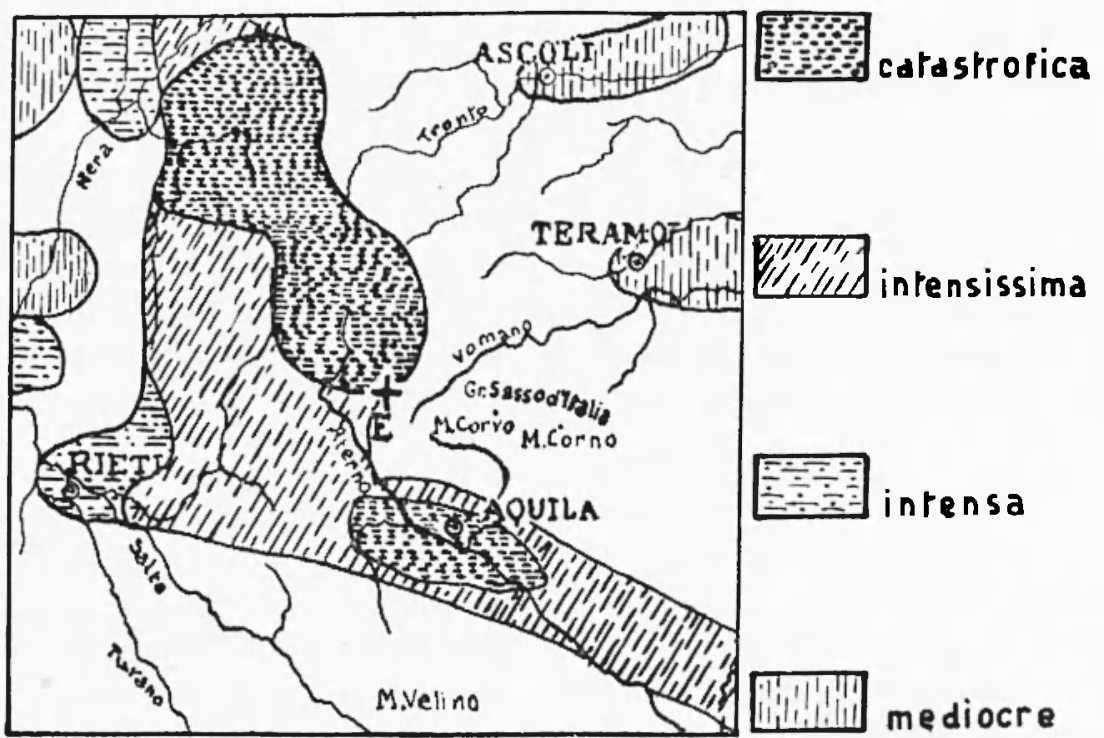

Fig. 7

proprio nel Gran Sasso (in corrispondenza del Como Grande). Largamente rappresentato nella zona è anche il cretaceo, sia nella formazione calcarea compatta che in quella a facies-scaglia (Corno Piccolo, Corno Grande, Monte Portella, Pizzo Cefalone, Monte Camicia...). Pure uno straordinario sviluppo hanno l'eocene e l'oligocene che costituiscono quasi il mantello superiore generale del gruppo montuoso del Gran Sasso (Monte Corvo, Punta Malecoste, Pizzo lntcrmesoli, Monte S. Franco...).

Intense forze orogeniche dehbono avere indubhiamente agito in queste zone: lo attestano i numerosi casi di sollevamenti, arricciamenti e addirittura rovesciamenti che presentano in alcuni punti gli strati eocenici. A Pizzo Intermesoli l'eocene raggiunge una altezza di più 


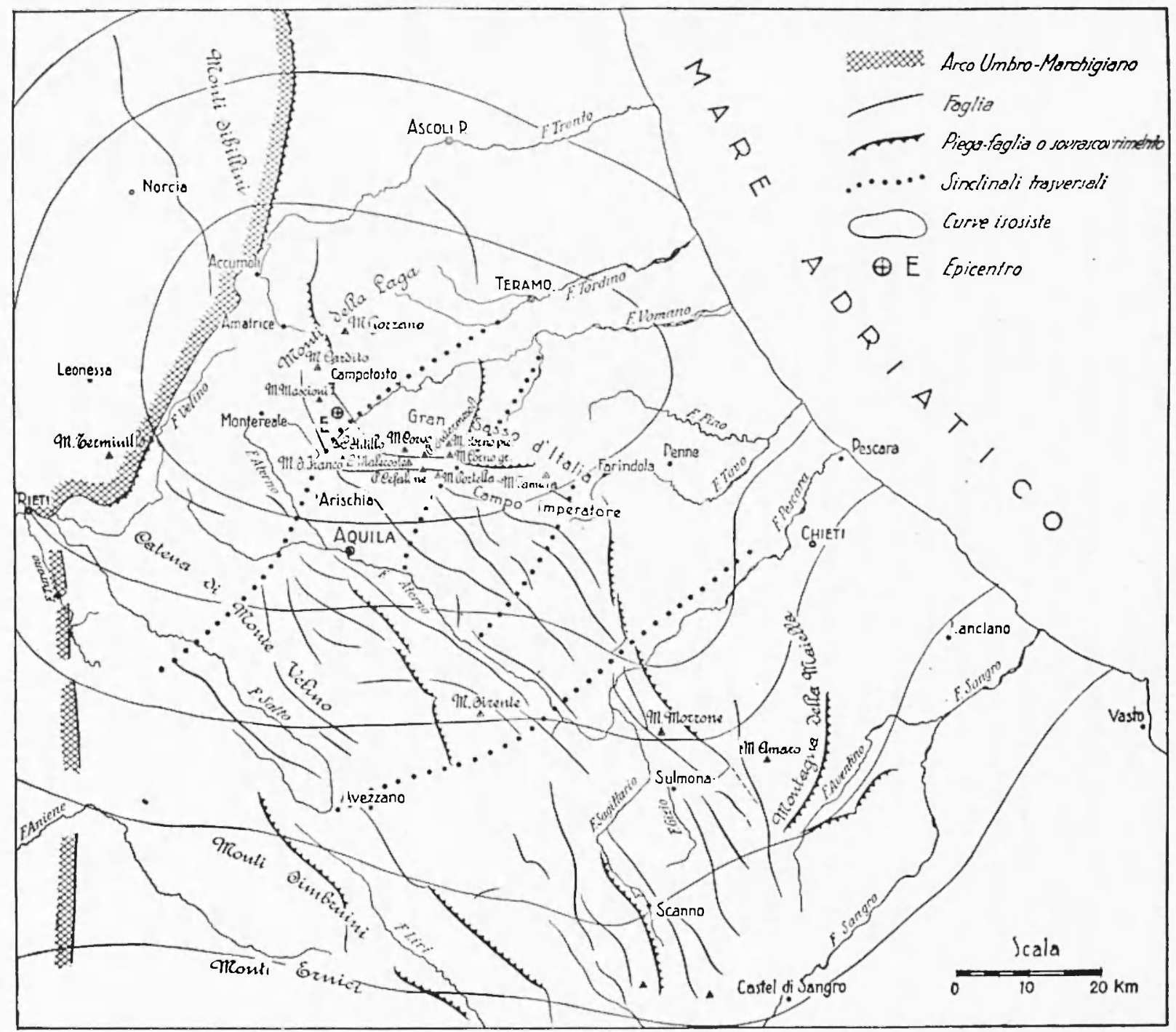

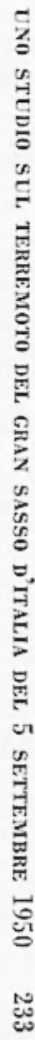

Fig. 8 
di 2600 metri. Woterole anche la potenza dell'eocene a Monte S. Franco (appena a Sud-Est dell'epicentro) dove però gli strati non sembrano presentare deiormazioni crogenetiche, mentre a soli $8 \mathrm{~km}$ di distanza (Cima Malecoste) gli strati sono fortemente raddrizzali e contorti. Una spessa coltre calcareo-marnosa del miocene (langhiano, elveziano, tortoniano) forma degli strati laterali specie sui versanti oricutali di Monte S. Franco, Monte Corvo, Pizzo Intermesoli, Corno Piccolo, e copre più o meno vaste zone dell'Appennino Alruzzese. Grande sviluppo superficiale hamno i terreni plastici posteclveziani: la molassa pontica, infatti, copre grandissime distese -- quasi a semicerchio - intorno ai massicei più elevati della catena Appenninica, elevandosi in qualche punto addirittura a zona nonluosa (Giaccio Porcelli, Monte Carditol: anche la gronda sinclinale del Vomano is costituita prevalentemente di molassa pontica.

La struttura stratigrafica della zona viene complelala da vasti depositi quaternari (detriti, alluvioni...) che di solito colmano zone di depressione (Campo Imperatore una delle più vaste; più piccole nei dintorni di Montereale, Campotosto, Assergi, Conche Aquilane). E al limite meridionale della depressione quaternaria di Campotosto che si trova l'epicentro, sui terreni plastici della molassa pontica, a pochi chilometri dai terreni semi-rigidi e rigidi dell'elveziano. La sua posizione, nei riguardi degrli assi tetlonici dell'Appennino Abruzzese quasi al punto in cui si incontrano la direthice setlentrionale del sistema abruzzese (Monte S. Franco, Monte Corro, Corno Piceolo) con quella trasversale costituita dalla sinclinale del Vomano, e con la diretrice appenninica orientala presso a poco lungo la retla MonterealeArischia.

Di importanza eccezionale è lo stile tettonico dei Monti della Laga che costituiscono il prolungamento naturale della Catena del Gran Sasso.

Tre elementi di direzione appenninica si distinguono nell'insieme di questa tettonica (v. fig. 9).

1) Un mradino inferiore occidentale, la cui parte settentrionale (Accumoli) - pur non essendo stata studiata particolaregriatamente - si può supporre relatiramente complessa per l'immediata ricinanza dell'arco Umbro-Marchigiano. Su questo gradino si trova il bacino di Amatrice che sembra rispondere ad una struttura sinclinale - m. 900 - , ma a Sud si sollera fino a 1600 metri (Monte Mascioni) e sembra proseguire con una anticlinale di qualche chilometro, la quale però presenta delle ondulazioni di cui il lago artificiale di Campo- 
tosto occupa qualche cavita. Nella parte occidentale, questo gradino scende con una fagrlia nettissima sul bacino di Montereale: questa faglia si prolunga a sud-est dove taglia il Monte S. Franco. Sul gratdino in esame si rilerano due sistemi tettonici poiché is stato appurato che sul Colle Spitillo, dalla molassa pontica emergono obliquamente dei calcari cocenici, che prolungano quelli del Gran Sasso.

2) Un secondo gradino orientale, sopraelevato rispetto al primo fino a 24.50 metri (Monte Gorzano), è costituito da un ammasso di calcari arenari e di molassa miocenica.

3) Tra l'uno e l'altro gradino corre una lunga fratiura rlye li dislivella hruscamente, con un rigetto di circa 1000 metri rerso Cam-

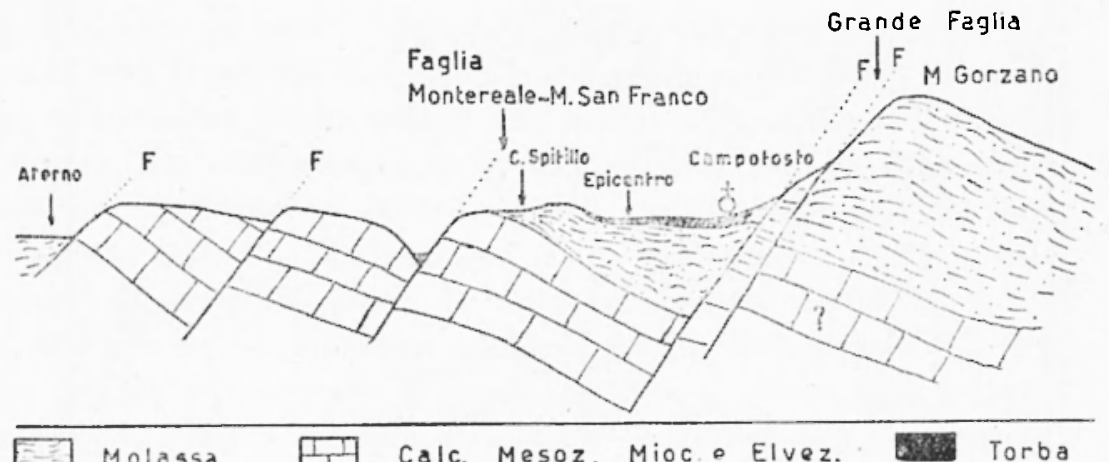

Fig. 9

potosto. Questa faglia gigantesca i normale a sud: ma a nord, a desta di Amatrice, si rovescia e si trasforma in una piega. A sud questa grande faglia continua al di lá del Vomano, raceordandosi con la tet. tonica di asse alıruzzese.

A sud dei Monti della Lag̣a passa la grronda radiale del Fomano. A Nord di questa gronda, tra le due faglie, che per un tratto corrono parallelamente ad una distanza inferiore ai $10 \mathrm{~km}$, cade l'epicentro del no:tro terremoto, quasi addossato alla faglia maggiore, la quale, d'altronde sembra essere tuttora attiva. Non è da escludere che il terremoto del 5 settembre alblia potuto contribuire all'approfondimento di questa grande faglia. E del resto le numerosissime faglie che tagliuzzano tutta la zona, tanto da giustificarne la denominazione di "Campo fagliato dell'Aterno ", datogli da Benco, quasi con certezza soato collegate intimamente con la sismicita del luogro, efletto di questa; al contrario di quanto sembra avenire nelle $A l_{p}$ i dove invece sono le faglie a provocare i terremoti. 
Impulso iniziale e natura della scossa. - Se ci soffermiamo ad analizzare per un momento la rete delle isosiste, possiamo vedere che quella anomalia già segnalata precedentemente nella forma delle curve, ha un carattere di deformazione preferenziale: tutte le isosiste, infatti, presentano un aumento di curvatura a sud-est dell'epicentro. Se si tracciasse una linea raccordante i punti di massima curvatura,

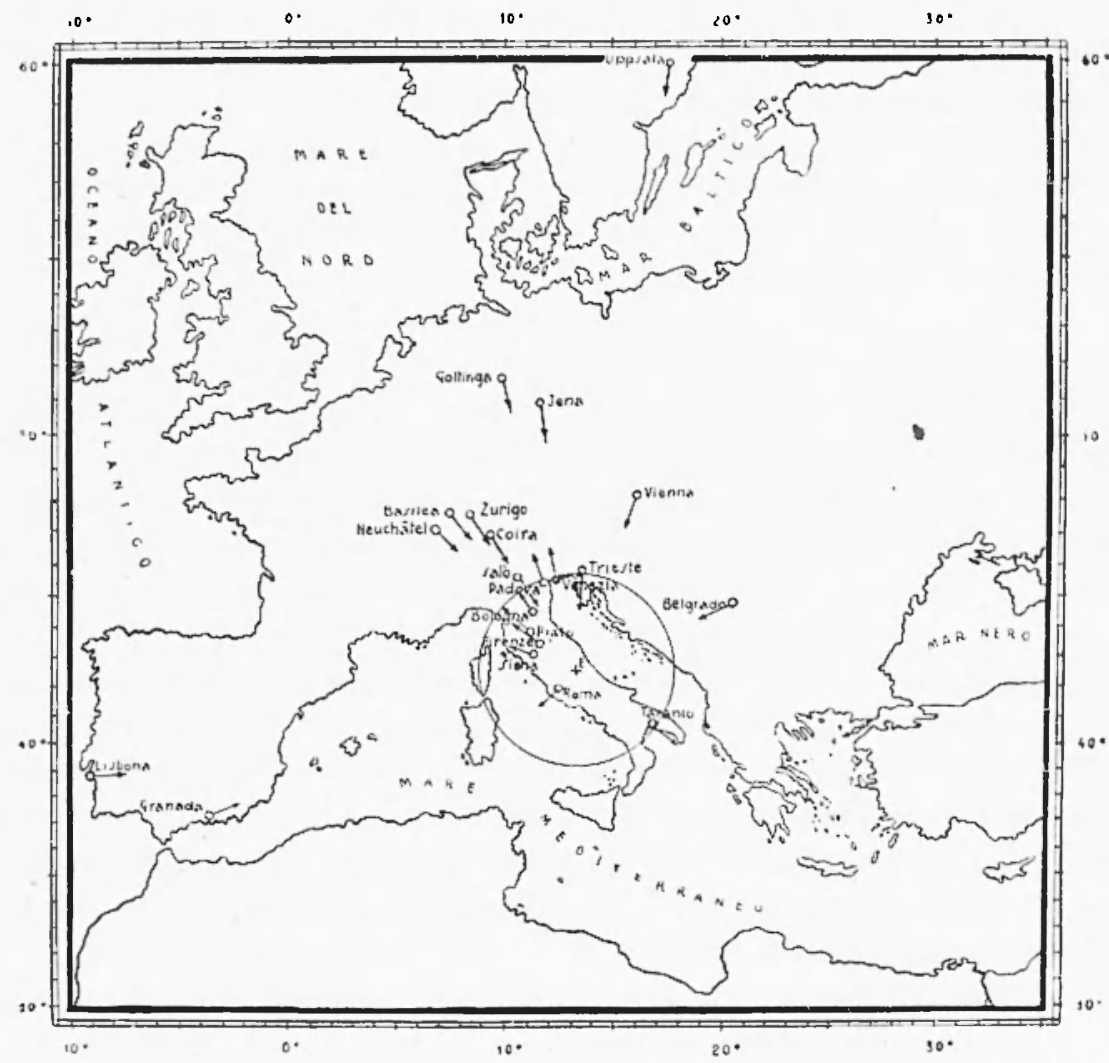

Fig. 10

questa linea risultereble orientata all'incirca come le direttrici del sistema appenninico, perpendicolare quasi a tutte le sinclinali trasversali della zona. L'orientamento di questa linea si accorderebbe inoltre con quello delle tante farlie, piccole e grandi, vecchie e giovari, che fanno dell'Appennino abruzzese un mosaico di compartimenti fagliati (vedi fig. 8 ).

Non possiamo azzardarci a trarre delle conclusioni. Tuttavia si può ritenere certo che l'orogenesi si è prolungata fino ad oggi. Il greo- 
logo tedesco Suter ritiene che $\left({ }^{12}\right)$ non sia improbalsile un ulteriore sollevamento post-glaciale. (Nella zona ḋ credenza popolare che il massiccio del Gran Sasso si sollevi di un metro l'anno). Noi da parte nostra possiamo asserire che il terremoto del 5 settemlyre 1950 è dovuto proprio ad un sollevamento di strati.

Infatti. dallo spoglio delle registrazioni albliamo potuto rilevare, per molte stazioni, la natura dell'impulso iniziale (vedi tabella 2). Le onde longitudinali sono emerse, nelle stazioni piì vicine all'epicentro e in tulti gli aximut, come compressioni: Padova, a $341 \mathrm{~km}$ dall'epicentro, presenta ancora una compressione, mentre a Trieste, di soli $8 \mathrm{~km}$ più lontana, l'onda compare come dilatazione, e dilatazioni compaiono pure in tutte le stazioni a magrgior distanza, qualunque sia il loro azimut. Il cerchio che separa la zona delle compressioni da quella delle dilatazioni risulta quindi ben individuato: il suo raggio è di $345 \mathrm{~km}$ ca. La distribuzione delle compressioni e delle dilatazioni (messa bene in evidenza dalla carta della fig. 10) ublidisce ad uno degli schemi tipici fondamentali: si tratta, evidentemente, di un sollevamento di strati. Né questo è l'unico esempio. Lo studio dei terremoti dell'Appennino tosco-romagnolo (5), della Garfagnana ( $\left.{ }^{6}\right)$, del Monte Amiata $\left({ }^{13}\right)$, avevano già messo in luce il carattere di un sollevimento di strati. L'esempio attuale può costituire una conferma e forse non $\delta$ troppo azzardato supporre che il fenomeno interessi l'intera catena montuosa dell'Appennino, dato che il terremoto in esame ha il suo fuoco presso la costa orientale, mentre i precedenti interessavano quella occidentale. La geologia conforta tali ipotesi con altri esempi: l'esame delle fronti di alcune farlie attestano infatti dei sollevamenti (1.4), quali ad esempio quello del Sirente e quello della catena del Gran Sasso verso Campo Imperatore.

$$
\text { Roma - Istituto Nazionale di Geofisica - Aprile } 1951 .
$$

\section{RIASSUNTO}

Nol presente lavoro si espongono $i$ risultati della prima parte di uno studio sul terremoto del Gran Sasso d'Italia avenuto il 5 settembre 1950. Si riportano le notizie macrosismiche, e viene tracciata la rete delle isosiste. Calcolata la magnitudo, lenergia, l'epicentro in coordinate ortogonali o geografiche, le velocità delle $\mathrm{Pg}, \mathrm{Pn}, \mathrm{S}_{\mathrm{g}}, \mathrm{Sn}$ con le relative dromocrone, il tempo origine, si trova infine che la 
profondità ipocentrale è trascurabile (dell'ordine di qualche kim). Trattandosi di un terremoto superficiale si ritiene opportuno dare alcune notizie geologiche della zona epicentrale. L'epicentro è molto prossimo ad una grande faglia (attiva) che va dal Nord di Amatrice fino a sud del Gran Sasso. Lo spoglio delle registrazioni indica che la scossa è dovuta ad un sollevamento di strati. Questo concorda con le ipotesi dei geologi $i$ quali suppongono che l'orogenesi dell'Appennino sia ancora in atto.

\section{BIBLIOGRAFIA}

(1) SiEderg A., Die Erdbeben. Hundbook der Geophysik. Band IV, 2.

(2) Di Filippo D. - Marcellı L., Magnitudo ed energia dei terremoti in Italix. Annali di Geofisica. Vol. III, n. 3.

(3) Cator P., Determinazione delle coordinate epicentrali di un terremoto ad origine vicina con $i$ tempi delle onde longitudinali e trasversali dirette. La Ricerca Seientifica. Anno 12, n. 4.

(4) Calo1 P., Caratteristiche sismiche fondamentali dellEuropa Centrale. Boll. Soc. Sismol. Italiana. Vol. XL, n. 3-4.

(5) Calor P., Caratteristiche sismiche dell'Appennino tosco-romagnolo. La Ricerca Scientifica. Anno XI, n. 4.

(6) Rosini E., Il terremoto della Garfagnana del 15 ottobre 1939. La Ricerca Scientifica. Anno XI, n. 7.8.

(7) Di Filippo D., Il terremoto di Teramo del 29-1-1943. Annali di Geofisica, 1949, vol. II, n. 2 .

(8) Wanti K., On the travel time of Earthquake Traves, Part II. Geophysical Magazine, vol. 7, 1933, pag. 101-111.

(9) Vicexte Ingidada Ons, Calcul des coordonnes du foyer seismique. Travaux Scientifiques, 1927, fasc. 5, serie A, pag. 23.

(10) Sacco F., Il gruppo del Gran Sasso d'Italia. Reale Accademia delle Scienze di Torino (Anno 1907-1908).

(11) Demanceor J., L'arc abruzzais externe - étude tectonique. La Ricerca Scientifica, 1951 (in corso di stampa).

(1-) Suter K., Die eiszeitliche Vergletscherung des Zentralappennins. Viertljahesschrift d. Naturforsch. Ges. Zürich, 1939.

(13) DI FiLIPpo D, Il terremoto del Monte Amiata del 19-VI-19:40. La Ricerca Scientifica, 7-8-9. Luglio-settembre 1941.

(14) Demangeot J., Tettonica degli Appennini del G. Sasso d'Italia. Contributi di Scienze Geologiche. Supplemento a La Ricerca Scientifica, 1950.

Gutenderc B., Theorie der Erdbcbenwellen; Beobachtungen. Handbook der Geophysik. Vol. IV.

Conran V. Das Schucudorfor Beben vom \& Oktober 1927. Gerlands Beitrïge zur Geophysik, XX, 1928.

Grife H., Das Norltiroler Beben vom 8 Oktober 1930. I Teil. Zeitschrift fïr Geophysik, VIII, 1932. 
Genevors G., Il terremolo di Derula del 3 novembre 1941. Boll. Soc. Sism. Italiana, XL, 1912, 1, 2.

Gionci M., Il terremoto del Monte Amiata del 16 oftobre 1940. Rie. Scient. 1941, n. 11.

Valle P. E., Contributo allo studio delle caratteristiche sismiche del Mediterraneo centro-orientale. Annali di Geofisica. Vol. I. n. 2 (19.8).

Catol P, Il terremolo adrintico del 30 novembre 1934. Boll. Soc. Sism. Ital. Vol. XXXV, fase. 3.t.

Calor P., Ricerche su terremoti ad origine vicina. íScosse del Cansiglio). Ottobre 1936. La Ric. Scient. (1933). Vol. II. 7.8.

Calor P., Studio microsismico del terremoto delle Prealpi Carniche. 8 giugno 1934. Boll. Comit. Geod. Geofis. del C. N. R. anno V (1935).

Di Filir'ro D., Il terremoto di Cernara di Roma dell's settembre 1941. Boll. Soc. Sism. Ital. Vol. XI, n. 1.2 (1912). 